\title{
QUERATOSIS ACTÍNICA Y SUS TRATAMIENTOS EN LOS ÚLTIMOS 4 AÑOS: UNA REVISIÓN
}

\author{
ACTINIC KERATOSIS AND ITS TREATMENTS IN THE LAST 4 YEARS: A REVIEW
}

\author{
Him Rios, Genesis Estefania*; Campos Herrera, Francisco Alonso† \\ *Estudiante del IX Semestre de Medicina, Universidad de Panamá, Ciudad de Panamá \\ †Médico Interno de segunda categoría, Complejo Hospitalario "Dr. Arnulfo Arias Madrid", Ciudad de Panamá
}

Recibido: 22 de mayo del 2020

Aceptado: 7 de septiembre del 2020

Him Rios G, Campos Herrera F. Queratosis actínica y sus tratamientos en los últimos 4 años: una revisión. Rev méd cient. 2020; Volumen 33(1):

página 89-96. DOI: $10.37416 /$ rmc.v33i1.563

\section{RESUMEN}

Antecedentes: Las queratosis actínicas son lesiones premalignas que pueden progresar a carcinoma espinocelular y se caracterizan por una proliferación de queratinocitos atípicos. Son pápulas eritematosas, descamativas que encontramos principalmente en áreas expuestas al sol. Es un problema frecuente en la consulta.

Objetivo: Recopilar información acerca del tratamiento de las queratosis actínicas en los últimos cuatro años, con el fin de ofrecerla de manera ordenada y sintetizada a todo estudiante, médico de atención primaria o secundaria que esté interesado en el tema.

Diagnóstico: Se puede realizar por medio de un examen clínico o histopatología, pero también hay otras opciones disponibles en centros dermatológicos especializados.

Tratamiento: Varía dependiendo del paciente, sus preferencias, sus comorbilidades; y de la lesión, si está aislada o se abarca un campo, el grado de la lesión, su localización, la cantidad de lesiones y tratamientos previos. En general, los podemos dividir en dos grupos: tratamientos para lesiones aisladas, como crioterapia, electrocirugía, legrado; y tratamientos para campo de cancerización, como 5fluorouracilo, terapia fotodinámica, imiquimod, diclofenaco y mebutato de ingenol.

Conclusiones: Al momento de tratar estas lesiones disponemos de varias opciones. Al reconocer la mejor para nuestro paciente y su correcto uso obtendremos mejores resultados y menor cantidad de recidivas.

\section{ABSTRACT}

Background: Actinic keratoses are premalignant lesions that can progress to squamous cell carcinoma and are characterized by a proliferation of atypical keratinocytes. They are erythematous scaly papules that are found mainly in areas exposed to the sun. It is frequent in the consult.

Objective: To collect information about the treatment of actinic keratoses in the last four years, in order to offer it in an orderly and synthesized way to every student and primary or secondary care physician who is interested in this subject.

Diagnosis: It can be done through a clinical examination or histopathology, but there are also other options available in specialized dermatology centers.

Treatment: It varies depending on the patient's preferences and comorbidities; the type of lesion, if it is isolated or is a field, the degree, its location, the number of the lesions and previous treatments. In general, we can divide them in two groups: treatments for isolated lesions, such as cryotherapy, electrosurgery, curettage; and cancer-related treatments, such as 5-fluorouracil, photodynamic therapy, imiquimod, diclofenac and ingenol mebutate.

Conclusion: At the time of treating these lesions we have several options. By recognizing the best option of treatment for our patient and its correct use, we will obtain better results and fewer recurrences. 


\section{INTRODUCCIÓN}

La queratosis actínica o queratosis solar es una de las lesiones cutáneas premalignas más importantes y se debe a la proliferación de queratinocitos atípicos. Esta lesión puede progresar a un carcinoma espinocelular. ${ }^{1}$ Es frecuente en la consulta de atención primaria y representa el 5-6\% del total de los pacientes atendidos en dermatología. ${ }^{2}$ En un estudio realizado en Panamá con 108 pacientes, 4.63\% presentaban queratosis actínica, tomando en cuenta que la edad media de la población era 47 años y que la mayoría era fototipo IV y $\vee$ de Fitzpatrick. $^{3}$

El cáncer de piel es el sexto más frecuente en Panamá y ha ido aumentando con los años; los últimos datos señalan que en el 2018 se registraron 219 casos en el Instituto Oncológico Nacional, siendo la población adulta mayor masculina la más afectada. ${ }^{4}$ El cáncer espinocelular es el segundo cáncer de piel más frecuente y el $60 \%$ se desarrolla a partir de una queratosis actínica, pero la probabilidad de que una queratosis actínica individual progrese a cáncer es baja. Este riesgo aumenta si aumenta el número de queratosis actínicas en la misma zona. ${ }^{2}$

\section{ETIOLOGÍA}

La queratosis actínica es el resultado de la exposición crónica a la radiación ultravioleta ${ }^{5}$ (Ver Tabla 1 ); por lo que es más común encontrarla en zonas expuestas como la cara, cuero cabelludo, orejas, cuello, zona del escote, antebrazos, dorso de las manos y piernas de las mujeres. ${ }^{1}$ Lo primero que se describe son cambios en la capa basal de la epidermis interfolicular. Además, estas lesiones se asocian a supresión del gen p53. ${ }^{6}$

Tabla1. Factores de riesgo asociados al desarrollo de queratosis actínica

\begin{tabular}{l} 
Factores de riesgo asociados al desarrollo de queratosis \\
actínica \\
\hline Exposición acumulada a la luz ultravioleta \\
Piel blanca \\
Edad avanzada \\
Genodermatosis (epidermolisis, xeroderma) \\
Inflamación crónica \\
Exposición a sustancias tóxicas (arsénico, alquitrán) \\
Inmunosupresión \\
Infección por el virus del papiloma humano \\
\hline Fuente: Actinic Keratosis and Cutaneous Squamous Cell Carcinoma. \\
Dtsch Arztebl Int. 20197
\end{tabular}

\section{PRESENTACIÓN CLÍNICA}

Las queratosis actínicas usualmente son asíntomáticas, pero pueden causar dolor o prurito. ${ }^{5}$ Son pápulas eritematosas, descamativas, a veces hiperqueratósicas que suelen medir menos de $1 \mathrm{~cm}$, pero al confluir pueden formar placas de mayor tamaño. ${ }^{2}$ La piel alrededor puede estar gruesa amarillenta, con hipopigmentación o hiperpigmentación, telangectasias y resequedad. Hay diversas manifestaciones de estas lesiones (Ver Tabla 2) y cuando la lesión se presenta en los labios se conoce como queilitis actínica. ${ }^{1}$

Tabla 2. Variantes clínicas de la queratosis actínica

\begin{tabular}{|c|c|}
\hline Variantes & Descripción \\
\hline Clásica & $\begin{array}{l}\text { Pápulas eritematoescamosas de hasta } 2 \\
\mathrm{~cm} \text { de diámetro }\end{array}$ \\
\hline Hipertrófica & $\begin{array}{l}\text { Presentan alguna escama gruesa, } \\
\text { adherente y queratósica }\end{array}$ \\
\hline Atrófica & Lesiones eritematosas sin escama \\
\hline $\begin{array}{l}\text { Con cuerno } \\
\text { cutáneo }\end{array}$ & $\begin{array}{l}\text { Lesiones queratósicas elevadas que } \\
\text { semejan una espícula o cono }\end{array}$ \\
\hline Pigmentadas & $\begin{array}{l}\text { Pápulas hiperpigmentadas de hasta } 1.5 \\
\mathrm{~cm} \text { con escamas }\end{array}$ \\
\hline
\end{tabular}

Fuente: Modificado de Dermatología. 6ta ed. Ciudad de México: McGraw-Hill Interamericana; $2015^{1}$

\section{EVOLUCIÓN}

Las queratosis actínicas comienzan como lesiones apenas visibles y palpables que luego progresan a lesiones hiperqueratósicas más gruesas (Ver Tabla 3$)^{5}$. Estas lesiones también pueden remitir, persistir o progresar a carcinoma espinocelular con los años. Se dice que el $15-25 \%$ remiten en un año, pero que el $15 \%$ de estas pueden reaparecer. Los pacientes trasplantados que se exponen a inmunosupresión crónica tienen mayor riesgo de recurrencia luego de tratamiento. ${ }^{8}$

Tabla 3. Grados de las queratosis actínicas

\begin{tabular}{ll}
\hline $\begin{array}{l}\text { Grados de las } \\
\text { queratosis actínicas }\end{array}$ & \multicolumn{1}{c}{ Descripción } \\
\hline Grado 1 & $\begin{array}{l}\text { Leve; coloración rosada o grisásea } \\
\text { arenosa al tacto }\end{array}$ \\
Grado 2 & $\begin{array}{l}\text { Moderado; hiperqueratosis fácilmente } \\
\text { detectable } \\
\text { Grave; hipertrófica con queratina } \\
\text { espesa }\end{array}$ \\
\hline $\begin{array}{l}\text { Campo de } \\
\text { cancerización }\end{array}$ & $\begin{array}{l}\text { Área que abarca varias lesiones; } \\
\text { pueden ser de diferentes grados }\end{array}$ \\
\hline
\end{tabular}

Fuente: British Association of Dermatologists' guidelines for the care of patients with actinic keratosis 2017. Br J Dermatol. $2017^{5}$ 
Siempre se ha pensado que lesiones de grado 3 tienen un riesgo elevado de progresar a cáncer en comparación con grados menores, pero se ha demostrado que el espesor de las lesiones no se relaciona con el grado de displasia. ${ }^{9}$ También se señala que lesiones de grado 1 pueden progresar directamente a carcinoma espinocelular por medio de una vía diferenciada, y que es difícil determinar qué lesiones progresarán a cáncer. ${ }^{6}$ Algunas características que pueden sugerir riesgo de progresión a malignidad son: induración, sangrado, inflamación, diámetro mayor de $1 \mathrm{~cm}$, crecimiento rápido, ulceración, prurito, dolor, lesión recurrente o refractaria al tratamiento y queilitis actínica. $^{8}$

\section{DIAGNÓSTICO}

Los aspectos clínicos por sí solos muchas veces son insuficientes para diagnosticar queratosis actínica. El estudio histopatológico es considerado el estándar de oro, pero algunos inconvenientes son que es un estudio invasivo, solo representa una pequeña parte de la lesión y en la práctica no se les hace biopsia a todas las lesiones, solo a las que aparentan malignidad. Hay otras opciones no invasivas como la dermatoscopia, la tomografía de coherencia óptica y la microscopia de reflectancia confocal que permiten la evaluación de toda la lesión (Ver Figura 1). Las dos últimas opciones son muy buenas para evaluar las queratosis actínicas, pero su uso es limitado en centros dermatológicos especializados. ${ }^{10}$

Figura 1. Herramientas para el diagnóstico de la queratosis actínica

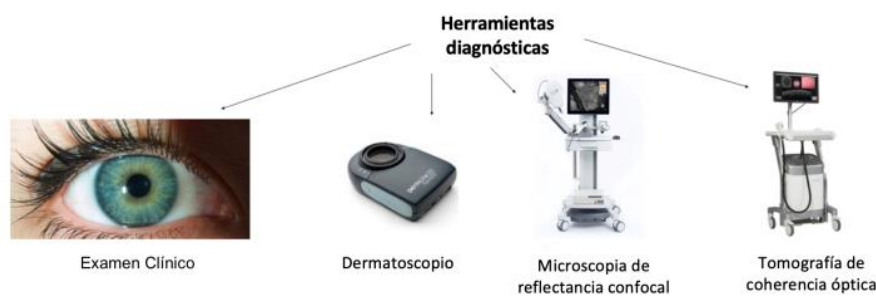

Fuente: Actinic Keratosis and Non-Invasive Diagnostic Techniques: An Update. Biomedicines. $2018^{10}$

El dermatoscopio tiene una sensibilidad del $98 \%$ y especificidad del 95\% para la detección de queratosis actínica. Las lesiones grado 1 se caracterizan por un patrón rojo en pseudored y escamas blancas discretas; las lesiones grado 2 son de fondo eritematoso donde se observan aperturas foliculares de color blanquecino o amarillento, conocido como patrón de fresa (Ver Figura 2); las lesiones grado 3 se caracterizan por aberturas foliculares agrandadas llenas de tapones queratósicos sobre un fondo escamoso y de color blanco amarillento o hiperqueratosis marcada en áreas blanco amarillentas sin estructura. ${ }^{10}$

Figura 2. Dermatoscopia de queratosis actínicas

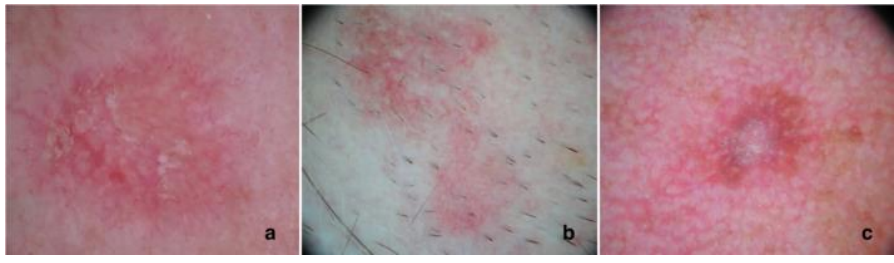

En la figura 2.a se observa el patrón de fresa con escamas; 2.b se observa un patrón de fresa sin escamas; 2.c se observa una lesión levemente pigmentada asociada a una pseudored eritematosa con escamas. Fuente: Actinic Keratosis and NonInvasive Diagnostic Techniques: An Update. Biomedicines. $2018^{10}$

El uso del dermatoscopio requiere un entrenamiento exhaustivo de su evaluador. En un estudio donde se comparaba el diagnóstico realizado a partir del examen físico implementando el uso del dermatoscopio con el telediagnóstico, se demostró que en las queratosis actínicas había una baja correlación entre los diagnósticos; por lo tanto, no es recomendable realizar telemedicina. En otros casos podría ser de ayuda en centros donde no cuentan con dermatólogos. ${ }^{11}$ Otro estudio demostró que la autoevaluación del paciente por medio de la observación normal es mejor que la autoevaluación con el dermatoscopio. ${ }^{12}$

Dentro del diagnóstico diferencial de las queratosis actínicas se encuentra la queratosis seborreica, dermatitis seborreica, psoriasis, lupus eritematoso discoide, verruga vírica, carcinoma basocelular superficial, léntigo solar, léntigo maligno melanoma, poroqueratosis y carcinoma epidermoide. ${ }^{2}$

\section{TRATAMIENTO}

Estas lesiones pueden ser evaluadas en la atención primaria donde se debe educar al paciente, considerar tratamiento y seguimiento. En algunos casos especiales se debe referir a un especialista (Ver Tabla 4). El manejo varía dependiendo de las preferencias del paciente, localización, cantidad de lesiones, extensión de las lesiones, tratamientos previos y hasta de la experiencia del médico (Ver Figura 3). ${ }^{5}$ La forma en la que el médico presenta la enfermedad y ofrece el tratamiento puede modificar la decisión del paciente. ${ }^{13}$ 
Tabla 4. Lesiones que se deben referir a un dermatólogo

\begin{tabular}{l}
\hline Lesiones que se deben referir a un dermatólogo \\
\hline Lesiones sin respuesta al tratamiento convencional \\
Lesiones múltiples o recurrentes difíciles de manejar \\
Pacientes con inmunosupresión crónica \\
Se sospecha malignidad
\end{tabular}

Fuente: British Association of Dermatologists' guidelines for the care of patients with actinic keratosis 2017. Br J Dermatol. $2017^{5}$

Muchas veces en la atención primaria no se evalúa correctamente este tipo de lesiones. La consulta se limita al motivo de consulta del paciente y no se realiza una exploración completa; a veces por falta de tiempo, falta de experticia, falta de conocimiento en las opciones terapéuticas, pero principalmente por no darle la importancia que amerita. Un mejor entrenamiento podría ayudar con el subdiagnóstico de estas lesiones y aminorar la carga en la atención secundaria. ${ }^{14}$

Figura 3. Tratamientos de la queratosis actínica

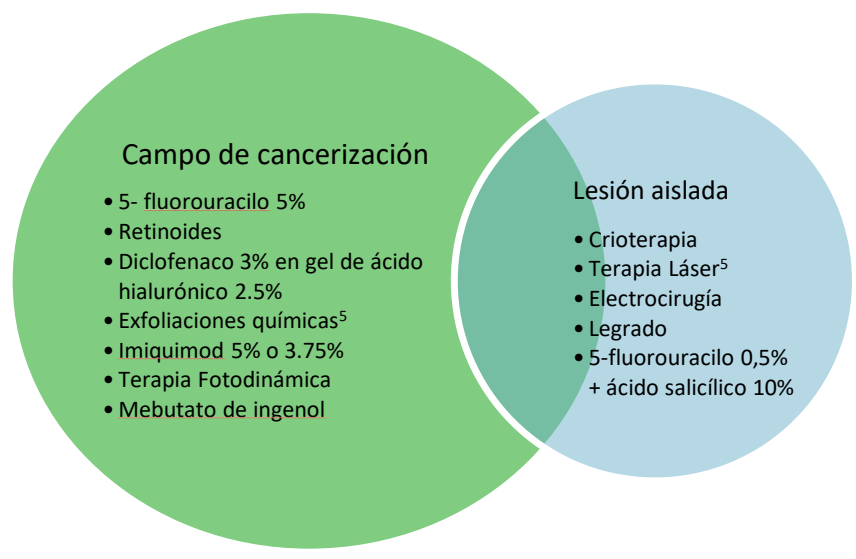

El tratamiento de lesiones aisladas es diferente al de un campo de cancerización; en este último el objetivo es tratar lesiones visibles y no visibles. En la práctica se combinan ambas terapias. Fuente: Queratosis actínica: Nuevo concepto y actualización terapéutica. Atención Primaria. $2017^{2}$ y British Association of Dermatologists' guidelines for the care of patients with actinic keratosis 2017. Br J Dermatol. $2017^{5}$

\section{Crioterapia}

La crioterapia es una de las terapias más antiguas y efectivas para la queratosis actínica. Consiste en la aplicación de un medio frío, generalmente nitrógeno líquido, a través de un dispensador en modo de aerosol sobre la lesión a tratar. ${ }^{2,5}$ Esto causa una necrosis por varios mecanismos; siendo una de las principales teorías el congelamiento de estructuras, tanto intracelulares como extracelulares, y la formación subsecuente de cristales, los que causan daño directo a organelas y a la membrana plasmática. ${ }^{15}$ Su ventaja radica en su fácil y rápida aplicación en lesiones únicas en un consultorio. La duración del tratamiento depende del número de dosis y el tiempo de aplicación; siempre tratando de evitar que exceda los 10 segundos. 2,5

Un estudio aleatorizado de 193 pacientes comparando crioterapia con terapia fotodinámica evidenció porcentajes de respuesta completa en $75 \%$ para crioterapia contra $69 \%$ en terapia fotodinámica. ${ }^{5}$ La diferencia fue más evidente cuando se trataba de lesiones más gruesas, 69\% contra 52\%. Entre los principales efectos adversos de la crioterapia están el dolor, la hipopigmentación y cicatrización, lo que lleva a los pacientes a preferir la terapia fotodinámica. ${ }^{5,16}$ Su principal limitación es en los campos de cancerización donde la terapia tópica ha ganado ventajas. La combinación de crioterapia con medicación tópica logra un mayor porcentaje de curación completa comparado con crioterapia sola según ocho estudios, pero no hubo diferencia significativa en curaciones parciales entre ambos tratamientos según 3 estudios. ${ }^{16}$

\section{Terapia Láser}

Muy efectiva logrando la eliminación de queratosis actínica. $90 \%$ responde a tratamiento y $10-15 \%$ recurren a los 6 meses. Hay dos tipos de láser: el ablativo, que elimina la epidermis y dermis superficial por medio de dióxido de carbono y erbio; y el no ablativo, que evapora o coagula pequeñas columnas de piel sin afectar el tejido circundante por medio de erbio fraccionado. Dentro de los efectos adversos están: dolor, inflamación, cambios en la pigmentación, retraso en la reparación de la piel. ${ }^{17}$

\section{Cirugía}

Al querer determinar o tener duda de si la lesión es de tipo invasiva, y más aún en lesiones hiperqueratósicas, se debe hacer por medio de un examen histológico una escisión con anestesia. Esta terapia se utiliza en lesiones asiladas, no en campos de cancerización. . $^{5,17}$

\section{Legrado}

Se recomienda en lesiones hiperqueratósicas o que son refractarias a otros tratamientos locales. Debe ir seguido de una electrodesecación. Se asocia a un alto riesgo de cicatrización. ${ }^{17}$ 


\section{Tratamientos tópicos}

El 5 Fluorouracilo al 5\% ha demostrado ser significativamente más efectivo que imiquimod, terapia fotodinámica de metil aminolevulinato y mebutato de ingenol; presentó menor cantidad de fracasos en el tratamiento, definiendo fracaso como una resolución menor del 75\% de la lesión a los 3 meses. ${ }^{18}$ Este tratamiento también funciona para prevenir a largo plazo la aparición de nuevas queratosis actínicas, aunque solo se evaluó en una población mayor. ${ }^{19} \mathrm{Su}$ uso combinado con calcipotriol refiere una mejoría del $82 \% .{ }^{20}$ El 5 Fluorouracilo al $0.5 \%$ se usa combinado con ácido salicílico, este último mejora la eficacia del primero (Ver Tabla 5). ${ }^{5}$

Tabla 5. Características de los tratamientos tópicos

\begin{tabular}{|c|c|c|c|}
\hline Medicamento & $\begin{array}{l}\text { Mecanismo de } \\
\text { acción }\end{array}$ & Efectos Adversos & $\begin{array}{l}\text { Pauta de } \\
\text { aplicación }\end{array}$ \\
\hline $\begin{array}{l}5- \\
\text { Fluorouracilo } \\
5 \%\end{array}$ & $\begin{array}{l}\text { Inhibición de la } \\
\text { timidilato sintasa }\end{array}$ & $\begin{array}{l}\text { Dolor, } \\
\text { enrojecimiento y } \\
\text { formación de } \\
\text { costras }\end{array}$ & $\begin{array}{l}2 \text { veces al día } \\
\text { por } 4 \\
\text { semanas }\end{array}$ \\
\hline $\begin{array}{l}\text { 5- } \\
\text { Fluorouracilo } \\
0.5 \% \text { + ácido } \\
\text { salicílico al } \\
10 \%\end{array}$ & $\begin{array}{l}\text { Inhibición de la } \\
\text { timidilato sintasa y } \\
\text { queratinolisis }\end{array}$ & Irritación & $\begin{array}{l}1 \text { aplicación } \\
\text { por día hasta } \\
\text { por } 12 \\
\text { semanas }\end{array}$ \\
\hline $\begin{array}{l}\text { Ácido } \\
\text { Retinoico }^{17}\end{array}$ & $\begin{array}{l}\text { Antioxidante, } \\
\text { reducción de } \\
\text { células dañadas }^{17}\end{array}$ & $\begin{array}{l}\text { Fotosensibilidad, } \\
\text { eritema, } \\
\text { erosiones, } \\
\text { prurito, dolor }{ }^{17}\end{array}$ & $\begin{array}{l}\text { No se utiliza } \\
\text { como } \\
\text { primera } \\
\text { opción } \\
\text { terapéutica }{ }^{17}\end{array}$ \\
\hline $\begin{array}{l}\text { Diclofenaco } \\
3 \% \text { en ácido } \\
\text { hialurónico } \\
2.5 \%\end{array}$ & $\begin{array}{l}\text { Inhibición de la } \\
\text { ciclooxigenasa }\end{array}$ & $\begin{array}{l}\text { Igual que los } \\
\text { anteriores, pero } \\
\text { más leve }\end{array}$ & $\begin{array}{l}2 \text { veces al día } \\
\text { por } 60-90 \\
\text { días }\end{array}$ \\
\hline $\begin{array}{l}\text { Imiquimod } \\
3.75 \%\end{array}$ & Inmunomodulador & $\begin{array}{l}\text { Eritema, costras, } \\
\text { úlceras, } \\
\text { erosiones, } \\
\text { síntomas } \\
\text { parecidos a la } \\
\text { gripe }\end{array}$ & $\begin{array}{l}1 \text { vez al día } \\
\text { por } 2 \\
\text { semanas/2 } \\
\text { semanas de } \\
\text { descanso/ } 1 \\
\text { vez al día por } \\
2 \text { semanas }\end{array}$ \\
\hline Imiquimod 5\% & Inmunomodulador & $\begin{array}{l}\text { Eritema, costras, } \\
\text { úlceras, } \\
\text { erosiones, } \\
\text { síntomas } \\
\text { pseudogripales }\end{array}$ & $\begin{array}{l}3 \text { veces por } \\
\text { semana por } 4 \\
\text { semanas/ } 4 \\
\text { semanas de } \\
\text { descanso/ } 3 \\
\text { veces por } \\
\text { semana por } 4 \\
\text { semanas }\end{array}$ \\
\hline
\end{tabular}

Fuente: British Association of Dermatologists' guidelines for the care of patients with actinic keratosis 2017. Br J Dermatol. $2017^{5}$ y Current therapies for actinic keratosis. Int. J. Dermatol. $2020^{17}$

Imiquimod se prefiere en lesiones no hipertróficas ni hiperqueratósicas, en pacientes inmunosuprimidos cuando no se puede realizar crioterapia y las demás opciones son menos apropiadas. Se aplica en la noche y se lava a las 8 horas. $^{5}$ Imiquimod al $5 \%$ se asocia a una mejor resolución de las lesiones y menor cantidad de recurrencias que con diclofenaco. La frecuencia en que las lesiones evolucionaban al siguiente grado o a carcinoma era menor. ${ }^{21}$

El gel de diclofenaco al 3\% con ácido hialurónico al 2.5\% en un $78 \%$ de los pacientes las lesiones resuelven más del $50 \%$, pero solo el $15 \%$ resuelve completamente; la mayoría no presenta efectos secundarios y los pacientes referían sentirse satisfechos en el $85 \%$ de los casos. ${ }^{22}$ El mecanismo de acción sigue siendo desconocido, pero se propone que es por la inhibición de la ciclooxigenasa con posterior angiogénesis y proliferación celular. Las lesiones han demostrado alteraciones en el metabolismo, función e infiltrados celulares, lo cual es normalizado por el diclofenaco; esto podría ayudar a entender el mecanismo de acción posteriormente. ${ }^{23}$

El mebutato de ingenol se utiliza en dos presentaciones; 150 $\mu \mathrm{g} / \mathrm{g}$ para la cara y cuero cabelludo, que se aplica por tres días; y $500 \mu \mathrm{g} / \mathrm{g}$ para extremidades y tronco (Ver Figura 4), que se aplica por dos días. ${ }^{5}$ Esto se asocia a una mejor adherencia en comparación con los demás tratamientos tópicos. ${ }^{24}$ En pacientes con el virus de inmunodeficiencia humana recibiendo terapia antirretroviral, mebutato de ingenol es eficaz, bien tolerado y se observó que interrumpe la fase latente del virus, aumentando su transcripción, pero sin activar una respuesta inmune o proinflamatoria. ${ }^{25}$ El desoxato de ingenol está en estudio fase II y se demostró que resuelve parcial y completamente las lesiones, es más efectivo en lesiones no hiperqueratósicas y no hipertróficas, ${ }^{26}$ y que al igual que el mebutato de ingenol hay buena adherencia al tratamiento y satisfacción por parte de los participantes. ${ }^{27}$

Figura 4. Resolución de queratosis actínica grado 2 tratada con mebutato de ingenol

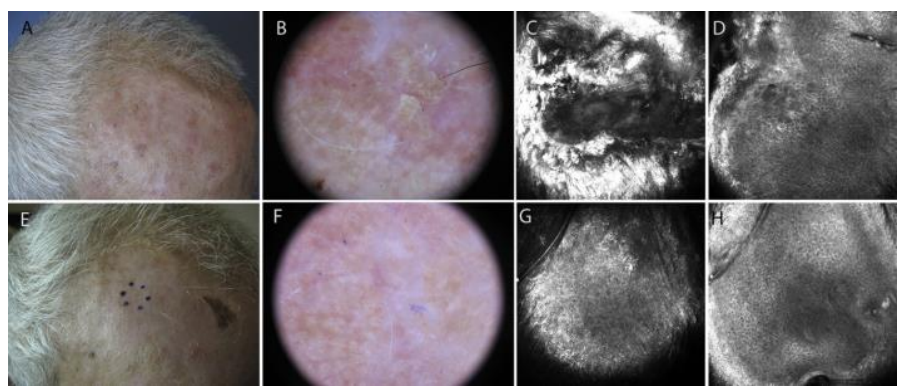

A: Apariencia clínica de la lesión. B: Imagen dermatoscópica mostrando fondo eritematoso con escamas amarillas y aberturas foliculares queratósicas. C: $750 \times 750 \mu \mathrm{m}$ imagen confocal de una marcada hiperqueratosis del estrato córneo. D: $750 \times 750 \mu \mathrm{m}$ imagen confocal de queratinocitos de diferentes 
tamaños y formas en el estrato espinoso. E-F: Apariencia clínica y dermatoscópica de la resolución de la lesión luego de mebutato de ingenol. G-H: 750-750 $\mu \mathrm{m}$ imagen confocal mostrando una piel de apariencia normal. Fuente: Identification of differentially expressed genes in actinic keratosis samples treated with ingenol mebutate gel. $2020^{28}$

\section{Exfoliaciones Químicas}

Recomendado como alternativa para queratosis actínicas extensas en el rostro. Consiste en la aplicación de agentes cáusticos, como: ácido tricloroacético, alfa hidroxiácidos, cloruro de zinc o ácido fenólico; provocando muerte celular a diferentes profundidades de la piel. 75\% de efectividad con 25 $35 \%$ de recaídas al año. Algunas complicaciones a considerar son: sobreinfección, inflamación, dolor, pigmentación anormal y riesgo de cicatrices. ${ }^{17}$

\section{Terapia Fotodinámica}

Esta terapia se basa en la aplicación de un fotosensibilizante tópico ocluido por 2 a 3 horas para luego iluminar la zona. Los fotosensibilizantes son captados selectivamente por células con alto índice mitótico que al ser iluminados se produce una reacción fototóxica que elimina las células. La irradiación con la luz puede ser dolorosa; requiriendo anestesia en ciertos casos. Cuando se realiza en lesiones hiperqueratósicas se debe realizar legrado previamente. ${ }^{2}$ Efectos adversos como eritema y costras pueden reducirse con el uso de yeso o fotoprotectores físicos. ${ }^{5}$ En pacientes trasplantados la terapia fotodinámica ha resultado ser más eficaz en comparación con el imiquimod, pero los pacientes no tienen preferencias entre los dos. ${ }^{29}$

Hay una variedad de luces disponibles; las fuentes de luz roja de espectro estrecho requieren menos tiempo de iluminación para dar tasas de respuestas altas. También se está realizando actualmente la terapia fotodinámica con la luz del día. Se aplica un protector solar, 15 minutos después la crema de metilaminolevulinato, que es el fotosensibilizante, y 30 minutos después el paciente se queda por 2 horas al aire libre. ${ }^{5}$ En un metaanálisis se observó que la terapia fotodinámica con luz de día no es inferior a la terapia fotodinámica convencional con respecto a las tasas de respuesta completa. Los pacientes preferían la terapia fotodinámica con luz de día, menos dolor, efectos adversos leves y resultados cosméticos excelentes. ${ }^{30}$

Dentro de los fotosensibilizantes están el ácido-5aminolevulínico (5-ALA), el metil-aminolevulinato (5-MAL) y ahora también BF-200 ALA que es un gel de nanoemulsión que contiene $7.8 \%$ de 5-ALA. Al comparar la terapia fotodinámica con luz de día con 5-MAL contra BF-200 ALA variables como la zona tratada, grado de la lesión, temperatura durante la fototerapia, tiempo atmosférico no mostraron diferencia significativa por lo que se concluye que la terapia con BF-200 ALA no es inferior a la terapia con 5-MAL. Además, las tasas de recurrencia eran menores con BF-200 ALA. ${ }^{31}$

\section{Otros}

Medicamentos sistémicos como los retinoides se pueden utilizar en pacientes inmunosuprimidos. Los agentes citotóxicos también se han considerado, pero hacen falta más estudios que lo respalden. Los retinoides tópicos han presentado un beneficio modesto. Otros medicamentos sistémicos sería los inhibidores de la ciclooxigenasa 2, pero estos solo han mostrado efecto sobre el carcinoma basocelular y el carcinoma espinocelular; no tanto en las queratosis actínicas. ${ }^{5}$

Se realizó un experimento con 11 pacientes para estudiar la utilidad de microondas en las queratosis actínicas. Las microondas mostraron una resolución del 15\%, pero en lesiones previamente tratadas la resolución era del $90 \%$ a los 120 días. Los pacientes que optaron por esta terapia mencionaron que era debido a su corto periodo de dolor. ${ }^{32}$

También se han desarrollado cremas dermatológicas con función protectora y reparadora de daño solar. Es una buena opción a nivel de atención primaria. Los principios activos varían según la casa farmacéutica. ${ }^{33}$

\section{CONCLUSIONES}

La exposición solar es acumulativa y sus consecuencias se evidencian en la edad adulta. La queratosis actínica es un ejemplo de ello. Educar a la población acerca de cómo realizar una correcta fotoprotección es primordial. También se le debe explicar al paciente que, aunque estas lesiones sean asintomáticas y puedan aparentar benignidad, siempre debe acudir a evaluación por un médico para evitar la progresión de la lesión.

Estudios en otros países señalan que los médicos generales no tienen claro su papel en estas afecciones y muchos no conocían todas las opciones terapéuticas que podían emplear. Sería bueno hacer un estudio similar en Panamá para evaluar la importancia que se le da a estas lesiones de manera rutinaria en la consulta, tratamientos empleados por los médicos 
generales y cuándo refieren al paciente. Muchas de las opciones expuestas solo pueden ser empleadas por dermatólogos. Sería excelente contar con una guía específicamente para atención primaria.

Para tratar estas lesiones disponemos de varias opciones, y al reconocer el mejor tratamiento para nuestro paciente y su uso correcto obtendremos mejores resultados y menor cantidad de recidivas. Antes de escoger el tratamiento siempre es de suma importancia conocer los cuidados al utilizarlo y al finalizar el tratamiento.

\section{BIBLIOGRAFÍA}

1. García C. Precáncer. En: Arenas Guzmán R. Dermatología. 6ta ed. Ciudad de México: McGraw-Hill Interamericana; 2015.p. 765-772.

2. Carmena-Ramón, R., Mateu-Puchades, A., SantosAlarcón, S., \& Lucas-Truyols, S. Queratosis actínica: Nuevo concepto y actualización terapéutica. Aten. Prim. [Internet]. 2017; 49(8):492-497. Disponible en: http://dx.doi.org/10.1016/j.aprim.2017.01.004

3. Ríos JM. Correlación entre las prácticas de exposición solar y el grado de fotodaño. Panamá. Mayo [Internet]. 2009. Rev méd cient. 2010;23(1):4-11. Disponible en: http://www.revistamedicocientifica.org/index.ph $\mathrm{p} / \mathrm{rmc} /$ article/viewFile/234/pdf 6

4. Instituto Oncológico Nacional "Dr. Juan Demóstenes Arosemena". Boletin del Departamento de Registro y Estadística de Salud [Internet]. 2018; Número 15. Disponible en: https://www.ion.gob.pa/site/wpcontent/uploads/2016/04/Boletin-Est.-2018-ofi.pdf

5. De Berker D, McGregor JM, Mohd Mustapa MF, Exton LS, Hughes BR. British Association of Dermatologists' guidelines for the care of patients with actinic keratosis 2017. $\mathrm{Br} J$ Dermatol [Internet]. 2017;176(1):20-43. Disponible en: https://onlinelibrary.wiley.com/doi/abs/10.1111/ bjd.15107

6. Fernandez M. From actinic keratosis to squamous cell carcinoma: pathophysiology revisited. J Eur Acad Dermatol Venereol [Internet]. 2017;31(2):5-7. Disponible en: http://doi.wiley.com/10.1111/jdv.14151

7. Gutzmer R, Wiegand S, Kölbl O, Wermker K, Heppt M, Berking C. Actinic Keratosis and Cutaneous Squamous Cell Carcinoma. Dtsch Arztebl Int. 2019;116(37):61628.

8. Actinic Keratosis [Internet]. DynaMed [citado 18 mayo 2020] Disponible en: https://www.dynamed.com/condition/actinickeratosis
9. Heerfordt IM, Nissen C V., Poulsen T, Philipsen PA, Wulf HC. Thickness of Actinic Keratosis Does Not Predict Dysplasia Severity or P53 Expression. Sci Rep [Internet]. 2016;6:1-6. Disponible en: http://dx.doi.org/10.1038/srep33952

10. Casari A, Chester J, Pellacani G. Actinic Keratosis and Non-Invasive Diagnostic Techniques: An Update. Biomedicines [Internet]. 2018;6(1):8. Disponible en: http://dx.doi.org/10.3390/biomedicines6010008

11. Santa-Vélez C, Rueda X, Pulido L, Pozzobón C, Sánchez $\mathrm{R}$, Acosta Á. Concordancia diagnóstica de la evaluación de imágenes digitales clínicas y dermatoscópicas versus el examen clínico de lesiones tumorales de piel. Rev la Asoc Colomb Dermatología y Cirugía Dermatológica [Internet]. 2018;26(2):94-105. Diponible en: https://doi.org/10.29176/2590843X.45

12. Janda M, Horsham C, Vagenas D, Loescher LJ, Gillespie $\mathrm{N}$, Koh $U$, et al. Accuracy of mobile digital teledermoscopy for skin self-examinations in adults at high risk of skin cancer: an open-label, randomised controlled trial. Lancet Digit Heal [Internet]. 2020;2(3):e129-37. Disponible en: http://dx.doi.org/10.1016/S2589-7500(20)30001-7

13. Berry K, Butt $M$, Kirby JS. Influence of information framing on patient decisions to treat actinic keratosis. JAMA Dermatology [Internet]. 2017;153(5):421-6. Disponible

en: http://archderm.jamanetwork.com/article.aspx?d oi=10.1001/jamadermatol.2016.5245

14. Noels $E$, Lugtenberg $M$, van Egmond S, Droger S, Buis $P$, Nijsten $T$, Wakkee $M$ Insight into the management of actinic keratosis: a qualitative interview study among general practitioners and dermatologists. Br J Dermatol. 2019 [Internet] ;181(1):96-104. Disponible en: https://onlinelibrary.wiley.com/doi/abs/10.1111/ bjd.17818

15. Tobón M, Franco V, Fierro E. Criocirugía. Rev Asoc Colomb Dermatol. [Internet]2014; 22 (4): 303-316 Disponible

en: https://revistasocolderma.org/sites/default/files/ criocirugia.pdf

16. Heppt M V., Steeb T, Ruzicka T, Berking C. Cryosurgery combined with topical interventions for actinic keratosis: a systematic review and meta-analysis. Br J Dermatol. [Internet] 2019;180(4):740-8. Disponible en: https://onlinelibrary.wiley.com/doi/abs/10.1111/ bjd. 17435

17. Jansen MHE, Kessels JPHM, Nelemans PJ, Kouloubis N, Arits AHMM, Van Pelt HPA, et al. Randomized trial of four treatment approaches for actinic keratosis. N Engl J Med [Internet]. 2019;380(10):935-46. Disponible en: http://www.nejm.org/doi/10.1056/NEJMoa18118 $\underline{50}$ 
18. Walker JL, Siegel JA, Sachar M, Pomerantz H, Chen SC, Swetter SM, et al. 5-Fluorouracil for Actinic Keratosis Treatment and Chemoprevention: A Randomized Controlled Trial. J Invest Dermatol [Internet]. 2017;137(6):1367-70. Disponible en: https://linkinghub.elsevier.com/retrieve/pii/S002 2202X17301057

19. García-Galaviz RA, Díaz-González José Manuel, CanoAguilar LE, Domínguez-Cherit J. Uso actual de la vitamina D en dermatología. Med Cutan Ibero Lat Am. [Internet]. 2019; 47(3):170-7. Disponible en: $\quad$ https://www.medigraphic.com/cgibin/new/resumen.cgi?IDARTICULO=91754

20. Dianzani C, Conforti C, Giuffrida R, Corneli P, di Meo N, Farinazzo $E$, et al. Current therapies for actinic keratosis. Int. J. Dermatol. [Internet]. 2020; 59: 677684. Disponible en: https://doi.org/10.1111/ijd.14767

21. Gollnick H, Dirschka T, Ostendorf R, Kerl H, Kunstfeld R. Long-term clinical outcomes of imiquimod $5 \%$ cream vs. diclofenac $3 \%$ gel for actinic keratosis on the face or scalp: a pooled analysis of two randomized controlled trials. J Eur Acad Dermatology Venereology [Internet]. 2020;34(1):82-9. Disponible en: https://onlinelibrary.wiley.com/doi/abs/10.1111/j dv.15868

22. Agata J, Valentina D, Teresa O, Giorgio C, Giacomo B, Matteo D, et al. The Relapse rate in patients with actinic keratosis treated with diclofenac sodium $3 \%$ gel. Int J Med Sci Clin Invent. [Internet]. 2019;6(2):4313-7. Disponible en: https://valleyinternational.net/index.php/ijmsci/articl e/view/1867

23. Singer K, Dettmer K, Unger $P$, Schönhammer G, Renner K, Peter K, et al. Topical Diclofenac Reprograms Metabolism and Immune Cell Infiltration in Actinic Keratosis. Front Oncol [Internet]. 2019;9:1-13. Disponible

en: https://www.frontiersin.org/article/10.3389/fonc. $\underline{\text { 2019.00605/full }}$

24. Norrlid H, Norlin JM, Holmstrup H, Malmberg I, Sartorius K, Thormann $\mathrm{H}$, et al. Patient-reported outcomes in topical field treatment of actinic keratosis in Swedish and Danish patients. J Dermatolog Treat [Internet]. 2018;29(1):68-73. Disponible en: https://www.tandfonline.com/doi/full/10.1080/0 9546634.2017.1329514

25. Jiang G, Maverakis E, Cheng MY, Elsheikh MM, Deleage C, Méndez-Lagares G, et al. Disruption of latent HIV in vivo during the clearance of actinic keratosis by ingenol mebutate. JCI Insight. [Internet] 2019;4(7):1-10. Disponible en: https://insight.jci.org/articles/view/126027

26. Siegel D, Tyring S, Nahm W, Osterdal M, Petersen A, Berman B. Three-day Field Treatment with Ingenol
Disoxate (LEO 43204) for Actinic Keratosis. A Phase II Trial. J Clin Aesthet Dermatol [Internet]. 2017; 10(12): 19-26. Disponible en: https://www.ncbi.nlm.nih.gov/pmc/articles/PMC577 4900/

27. Berman B, Tyring S, Nahm WK, et al. Three-day field treatment with ingenol disoxate (LEO 43204) for actinic keratosis: cosmetic outcomes and patient satisfaction from a phase 2 trial. J Clin Aesthet Dermatol [Internet]. 2017;10(11):26-32. Disponible en:

https://www.ncbi.nlm.nih.gov/pmc/articles/PMC577 4908/

28. Segura S, Gadea A, Nonell L, Andrades E, Sa S, Pujol R, et al. Identification of differentially expressed genes in actinic keratosis samples treated with ingenol mebutate gel. Plos One. [Internet]. 2020 15(5);1-20. Disponible

en: https://dx.plos.org/10.1371/journal.pone.023214 $\underline{6}$

29. Togsverd-Bo K, Halldin C, Sandberg C, Gonzalez H, Wennberg AM, Sørensen SS, et al. Photodynamic therapy is more effective than imiquimod for actinic keratosis in organ transplant recipients: a randomized intraindividual controlled trial. $\mathrm{Br} J$ Dermatol [Internet]. 2018;178(4):903-9. Disponible en: https://onlinelibrary.wiley.com/doi/abs/10.1111/ bjd.15884

30. Tomás-Velázquez A, Redondo P. De la terapia fotodinámica convencional a la terapia fotodinámica con luz de día en el tratamiento de las queratosis actínicas: revisión sistemática y metaanálisis. Actas Dermosifiliogr [Internet]. 2017;108(4):282-92. Disponible

en: https://linkinghub.elsevier.com/retrieve/pii/S000 1731016303507

31. Dirschka T, Ekanayake-Bohlig S, Dominicus R, Aschoff $R$, Herrera-Ceballos E, Botella-Estrada $R$, et al. A randomized, intraindividual, non-inferiority, Phase III study comparing daylight photodynamic therapy with BF-200 ALA gel and MAL cream for the treatment of actinic keratosis. J Eur Acad Dermatology Venereol [Internet]. 2019;33(2):288-97. Disponible en: http://doi.wiley.com/10.1111/jdv.15185

32. Jackson DN, Hogarth FJ, Sutherland D, Holmes EM, Donnan PT, Proby CM. A feasibility study of microwave therapy for precancerous actinic keratosis. $\mathrm{Br} \mathrm{J}$ Dermatol [Internet]. 2020 183(2);1-9. Disponible en: https://onlinelibrary.wiley.com/doi/abs/10.1111/ bjd.18935

33. Eryfotona AK-NMSC Fluid SPF100+. ISDIN [Internet]. 2020. Disponible en:

https://www.isdin.com/producto/eryfotona/aknmsc-fluid-spf100. 